\title{
Interpopulation spread of a parasitic B chromosome is unlikely through males in the grasshopper Eyprepocnemis plorans
}

\author{
María Inmaculada Manrique-Poyato ${ }^{1}$ Josefa Cabrero ${ }^{1}$ - María Dolores López-León ${ }^{1}$ • Francisco Perfectti ${ }^{1}$. \\ Ricardo Gómez ${ }^{2}$ - Juan Pedro M. Camacho ${ }^{1}$
}

Received: 10 May 2019 / Revised: 11 June 2019 / Accepted: 25 June 2019 / Published online: 8 July 2019

(C) The Author(s), under exclusive licence to The Genetics Society 2019

\begin{abstract}
The near-neutral model of B chromosome evolution predicts that population invasion is quite fast. To test this prediction, in 1994, we introduced males of the grasshopper Eyprepocnemis plorans from a B-carrying population into a B-lacking population and monitored the evolution of B-chromosome frequency up to 2013. We observed fluctuating very low B frequency across years but, remarkably, the B chromosome introduced (the B2 variant) was found up to 1996 only, whereas the B1 variant was present from 1996 onwards, presumably introduced by fishermen using E. plorans males as bait. Effective introgression of genetic material from the donor population was evidenced by the presence of a satellite DNA on autosome 9 (up to 1999) and the presence of one individual in 2006 showing an ISSR marker profile being highly similar to that found in the donor population. This indicated that the males introduced by us effectively mated with resident females, but donor genes rapidly decreased in frequency after this non-recurrent migration event. Taken together, our results indicated: (i) that the non-recurrent migration event had a slight, transient genetic effect on the recipient population, which was diluted in only a few generations; and (ii) that even with recurrent migration (forced by fishermen) the B chromosome failed to increase in frequency. Bearing in mind that B chromosomes in this species drive through females only, we hypothesize that B chromosomes most likely failed invasion in both migration events because the migrating sex shows no Bdrive.
\end{abstract}

\section{Introduction}

B (supernumerary or accessory) chromosomes usually behave as genomic parasites exerting deleterious effects on the individuals carrying them. They are maintained in natural populations at the expense of the so-called drive or accumulation mechanisms (Östergren 1945; Nur 1962; Hewitt 1976; Jones 1991). Alternatively, some B

Supplementary information The online version of this article (https:// doi.org/10.1038/s41437-019-0248-5) contains supplementary material, which is available to authorized users.

Juan Pedro M. Camacho

jpmcamac@ugr.es

1 Departamento de Genética, Facultad de Ciencias, Universidad de Granada, Avda. Fuentenueva s/n, 18071 Granada, Spain

2 Departamento de Ciencia y Tecnología Agroforestal, E.T.S. de Ingenieros Agrónomos, Universidad de Castilla La Mancha, 02071 Albacete, Spain chromosomes can be beneficial for their hosts, at least at low numbers, as predicted by the heterotic model (Darlington 1958; White 1973). Under this last model, a fast increase in B-chromosome frequency is expected due to phenotypic selection favouring B-carriers. However, the invasion by parasitic Bs triggers a coevolutionary dynamic that depends on a balance between (i) the intensity of drive, (ii) the negative phenotypic selection acting on B-carriers (due to B harmful effects and host intolerance), and (iii) the resistance offered by the receptor population in terms of drive suppression (Camacho et al. 1997). The higher complexity of coevolutionary interactions makes parasitic B invasions more unpredictable than heterotic ones.

One way to investigate the parasitic B chromosomes is to analyze the artificial invasion of $\mathrm{B}$ chromosomes within a B-lacking population after the release of individuals from a B-carrying population. This kind of experiment has only been previously performed in a natural population of the grasshopper Arcyptera fusca, where Gosálvez and LópezFernández (1984) released 50 males, for two consecutive 
years, and monitored B frequency for 7 years, with the final result of the complete elimination of the B chromosome.

The grasshopper Eyprepocnemis plorans shows B chromosomes which, in most cases analyzed, lack drive, with transmission rates being close to that predicted by Mendelian laws (López-León et al. 1992, 1993; Bakkali et al. 2002; Bakkali and Camacho 2004). However, other evidence points to the existence of drive through females, which was apparent after interpopulation controlled crosses (Herrera et al. 1996), and also for a new B variant (B24) that had recently invaded a natural population (Zurita et al. 1998). This led us to suggest that B chromosomes in $E$. plorans begin as parasitic elements, showing drive that is subsequently suppressed by the host genome. The existence of drive-suppressor genes was first theoretically suggested by Shaw (1984) and was empirically demonstrated by Shaw and Hewitt (1985) and Nur and Brett (1985). Our findings that a same E. plorans female transmits its B chromosome at a Mendelian rate when mated to a $0 \mathrm{~B}$ male from the same population, but it can drive its $\mathrm{B}$ if mated to a $0 \mathrm{~B}$ male from a B-lacking population (Herrera et al. 1996) suggested the existence of drive-suppressor genes in the B-carrying populations that were, however, absent (or rare) in Blacking populations. This led us to propose the near-neutral model of B chromosome evolution (Camacho et al. 1997), by which a $\mathrm{B}$ chromosome begins being parasitic (thus driving and being harmful for carriers) and this facilitates its rapid invasion (lasting only some tens of generations). Subsequent drive suppression leads the population to a near-neutral stage, which may be long lasting because B chromosomes reached high frequency and their elimination mainly depends on drift and how harmful they are at high numbers.

Almost all natural populations of the subspecies $E$. plorans hitherto analyzed carried B chromosomes (Camacho et al. 2003; López-León et al. 2008). The geographical range of this subspecies includes the whole CircumMediterranean region plus the Caucasus, the Arabian Peninsula, and the east of Africa. The species is of African origin, but other subspecies have been described in the south and west of Africa (Dirsh 1958). After the analysis of numerous samples from Spain, Morocco, Tunisia, Sicily, Greece, Turkey, Armenia, and Dagestan, the only B-lacking region found was in the headwaters of the Segura river and its tributaries in the east of the Iberian Peninsula (Cabrero et al. 1997), which is the location area for the two B-lacking populations where we performed the experiments described here.

We have registered two primary invasions, i.e., the first arrival of a B to a natural population, in Pollensa (Mallorca, Balearic Islands, Spain) (Riera et al. 2004) and Otívar (Granada, Spain) (Camacho et al. 2015), which indicate that primary B spread is still reaching new B-lacking populations in Spain, e.g., some river headwaters. This is consistent with the recent spread of the B1 chromosome across Western Mediterranean area (Cabrero et al. 2014) and with the extreme conservation of a $1510 \mathrm{bp} \mathrm{SCAR}$ DNA marker, being specific of B chromosomes, in populations as distant as Spain, Morocco, Greece, and Armenia (Muñoz-Pajares et al. 2011).

To unveil the dynamics of B chromosome invasions, in 1994, we started the artificial introduction of a B chromosome in two populations previously lacking it. We released 50 males from Salobreña (Granada, Spain), a population carrying the B2-type chromosome, into each of two populations at El Gallego and Vicorto (Albacete, Spain), where we had failed to find B chromosomes in a 1993 sample (Cabrero et al. 1997). The donor and receptor populations also differed for the presence of a $180 \mathrm{bp}$ satDNA on autosome 9 in the former but its absence in the latter, in coincidence with other populations at the Segura river basin (Cabrero et al. 2003).

We sampled the two populations in subsequent years to test the success of these experimental B invasions, and found B chromosomes only in El Gallego population. We scored the frequency of B chromosomes at this population in seven samples between 1996 and 2013, and performed double FISH to better characterize the type of B chromosomes found. In addition, we performed controlled crosses to three B-carrying females in 2000 and, in 2006, we analyzed ISSR (inter simple sequence repeat) markers in the donor and receptor populations, as well as in five other populations close to El Gallego, in order to search for some signs of nuclear gene introgression indicating whether the released males effectively mated with the resident females. The results showed the presence of some markers of the donor population indicating effective introgression into $\mathrm{El}$ Gallego, but there was a residually low frequency of B chromosomes during the analyzed period, with no significant tendency to increase and no signs of B invasion.

\section{Materials and methods}

\section{Population sampling}

Adult specimens of the grasshopper E. plorans were collected at eight Spanish populations: Salobreña (Granada province), Calasparra and Caravaca (Murcia), and El Gallego, Claras, Mundo, Socovos, and Vicorto (Albacete). No specific permits were required for most field studies, except the release of Salobreña males into El Gallego and Vicorto, where a special permit was obtained from the Consejería de Agricultura de la Junta de Castilla-La Mancha (Spain). Some locations sampled were privately owned but we obtained owners' permit for sampling. None of these 
locations was under environmental protection, and this field study did not involve endangered or protected species.

\section{Estimation of population size in El Gallego and release of males from Salobreña}

Prior to the release of B2-type-carrying males, we made an estimation of population size in one of the B-lacking populations by the triple catch method (Begon 1979), in order to use this estimate to calculate the starting B frequency after the release of Salobreña individuals. It was carried out in a field of about $2000 \mathrm{~m}^{2}$ located next to the Segura river in El Gallego (Albacete, Spain). This field harbored a variety of crops, with a predominance of maize and alfalfa. On September 30, 1994, we captured 71 males and 45 females which were marked with red nail varnish and released again into the population. Next day, we captured 60 males and 42 females, six and two of which, respectively, were recaptured as indicated by their nail varnish mark. All captured individuals were marked (included those recaptured) with pale pink nail varnish and were released. Next day, we captured 65 males and 44 females. Six males and two females carried the first-day mark, four males and one female carried the second-day mark, and a single male carried both marks. Before every capture period during the second and third days, we walked across the field in order to homogenize the population by forcing grasshoppers to jump. We then left the population rest for 15-20 min before beginning capture.

On October 5, 1994, we captured 100 males in the population of Salobreña (Granada, Spain), where the B2type frequency was temporarily stable and close to an average of 0.8 Bs per individual (Camacho et al. 1997), and released 50 of them in Vicorto and the other 50 in El Gallego (Albacete, Spain). We did not perform additional estimates of population size in El Gallego, and we think that it was almost similar in the different years of sampling, as we did not detect major deviations from the initial measurements.

\section{Monitoring B-chromosome frequency across years}

We sampled the El Gallego population in 1996, 1999, 2000, 2002, 2006, 2008, and 2013. In Vicorto, however, we decided stop sampling after failing to observe a single Bcarrying individual in 1996 and 1999. To analyze the evolution of B-chromosome frequency, we captured males and, occasionally, females. Males were anaesthetized with ethyl acetate vapors and testes were dissected out and fixed in freshly prepared 3:1 ethanol-acetic acid. Females were injected with $0.05 \%$ colchicine in insect saline $6 \mathrm{~h}$ prior to anesthesia and fixation of ovarioles in 3:1 ethanol-acetic acid. The presence and number of $\mathrm{B}$ chromosomes were

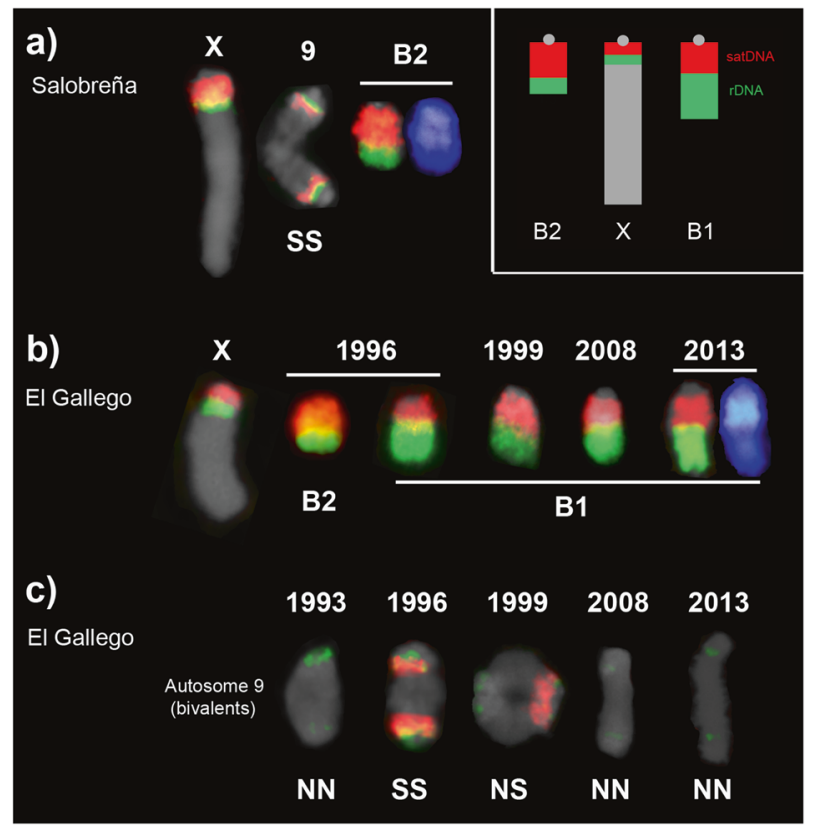

Fig. 1 Double FISH showing the distribution of $45 \mathrm{~S}$ ribosomal DNA (rDNA) (green) and the $180 \mathrm{bp}$ satellite DNA (satDNA) (red) on the B, $\mathrm{X}$ and autosome 9 in the donor (Salobreña) (a) and recipient (El Gallego) (b, c) populations. The inset on the upper right corner includes a schematic representation of $\mathrm{X}$ and $\mathrm{B}$ chromosomes showing that the $\mathrm{B} 2$ chromosome is about one third the size of the $\mathrm{X}$ chromosome and carries about double amount of satDNA than rDNA, whereas the $\mathrm{B} 1$ chromosome is about half the size of the $\mathrm{X}$ and carries about similar amounts of satDNA and rDNA (or more rDNA than satDNA in some cases). Note in El Gallego (b) the presence of the B1 and B2 variants in 1996, but only B1 in subsequent samples. In the donor population, autosome 9 was homozygous for the presence of satDNA (SS) (a), whereas in the recipient population (c) it lacked satDNA in 1993 (i.e., prior to the forced migration) ( $\mathrm{NN}=$ homozygous lacking the satDNA on autosome 9), whereas some individuals carried autosome 9 bivalents with satDNA in 1996 (SS $=$ homozygous) and 1999 (NS=heterozygous). The $\mathrm{X}$ chromosome is depicted as reference for $\mathrm{B}$ chromosome size. Note that the DAPI ${ }^{+}$and $\mathrm{DAPI}^{-}$patterns (shown in blue color) for B2 (a) and B1 (b) coincide with satDNA and rDNA locations, respectively

microscopically determined in squash preparations of testis tubules or ovarioles in lactopropionic orcein. We first characterized each B chromosome as B1 or B2 variants by means of the C-banding technique, which reveals a smaller distal region showing light C-banding in the latter (LópezLeón et al. 1993). For a more accurate characterization, we performed double fluorescent in situ hybridization (FISH) with two probes for the most abundant repetitive DNAs in E. plorans B chromosomes, i.e., ribosomal DNA (rDNA) and a 180 bp satellite DNA (satDNA). The FISH procedure employed was similar to that described in Cabrero et al. (2003). The B2 chromosome in Salobreña is about one third the size of the $\mathrm{X}$ chromosome, and has about double amount of satDNA than rDNA (Fig. 1), whereas the B chromosome found in populations nearby El Gallego (B1) is about half the size of the $\mathrm{X}$ and carries about similar amounts of 
satDNA and rDNA (Cabrero et al. 2014) (see also Fig. 1). All B-carrying males collected in El Gallego were analyzed by C-banding, and only some of them were also analyzed by double FISH. Comparison of B frequency across years was done by the $\mathrm{RxC}$ software (provided by G. Carmody, Ottawa, ON, Canada), which performs $\chi^{2}$ tests in contingency tables, with permutation, and calculates $P$ values by Monte Carlo methods, with 20 batches of 2500 replicates.

In the 2000 sample, we wanted to have the opportunity to analyze the transmission of the B chromosomes that appeared at low frequency. For this purpose, we vivisected all males and extracted several testis follicles through a small cut performed in the mid-dorsal abdomen, which were fixed and analyzed as explained above. No B-carrying males were observed. To analyze B presence in females, we maintained them alive for possible controlled crosses and followed a procedure to visualize $\mathrm{B}$ chromosomes in interphase nuclei of hemolymph (Cabrero et al. 2006). This revealed the presence of three B-carrying females, which were crossed with $\mathrm{OB}$ males from the same population.

As a positive control of the effective crossing of the Salobreña males with the El Gallego females, we performed both cytological and molecular analyses. The former consisted in the detection of cytological markers being characteristic of the Salobreña population in the samples collected at El Gallego after the release. For this purpose, we performed FISH analysis to test the presence of satDNA in the S9 autosome. The second approach consisted in analyzing ISSR markers in a postrelease sample from El Gallego, collected in 2006, and contemporary samples from Salobreña and five other populations geographically close to El Gallego (Claras, Socovos, Mundo, Caravaca and Calasparra). This allowed detecting signs of the effective introgression of Salobreña genes onto El Gallego population. All methods related to ISSR markers are described in detail in Manrique-Poyato et al. (2013a, 2013b). In brief, DNA was extracted with the kit GenElute Mammalian Genomic DNA miniprep (SIGMA), and DNA was quantified in a TBS-380 minifluorometer (Turner Biosystems) using Picogreen dye (QuantiT TM PicoGreen dsDNA Kit; Molecular Probes, Invitrogen). ISSR markers were obtained by polymerase chain reaction using the primers and conditions detailed in Table S12 of Manrique-Poyato et al. (2013a). Fragment sizes were determined with the HyperLadder II (Bioline) molecular-weight marker, and the presence or absence of each fragment was coded as 1 and 0 , respectively. Allele frequencies for ISSR markers were estimated by Bayesian methods, using the Hickory v1.1 software (Holsinger et al. 2002), bearing in mind their dominant nature, as explained in Manrique-Poyato et al. (2013b).

To identify the presence of El Gallego individuals showing a genetic profile resembling those in Salobreña, thus evidencing the introgression of nuclear markers coming from the Salobreña males, we used a Bayesian algorithm implemented in the Structure v2.3.1 software (Falush et al. 2007). We used the admixture model and the allele frequencies correlated model, without prior assumptions concerning the population. For each value of $\mathrm{K}$ (from 1 to 8 ), independent runs were made and, for each run, 100,000 iterations were carried out after a burn-in period of 50,000 iterations. To determine the number of genetically homogeneous groups $(\mathrm{K})$ that best fit the data, we followed Evanno et al. (2005), using the Structure Harvester website (Earl and VonHoldt 2012).

We investigated whether $\mathrm{B} 2$-chromosome transmission rate was consistent with the Mendelian one $(\mathrm{kB}=0.5)$ by means of the $Z$-test (López-León et al. 1992; Pardo et al. 1994; Lanzas et al. 2018) in the offspring of the three Bcarrying females mated with $0 \mathrm{~B}$ males. The number of eggs and embryos per pod and egg fertility were compared between B-carrying and B-lacking females by means of Student's $t$-tests. In addition, we analyzed meiotic transmission of the B1 chromosome in five 1B males collected in 2013 at El Gallego population, by means of FISH for rDNA or satDNA, which allowed to score the frequency of Bcarrying and B-lacking round (R) and elongated (E) spermatids (Cabrero et al. 2017). B transmission in these five males was tested by a goodness-of-fit $\chi^{2}$ test with expected 1:1 ratio for B-carrying and B-lacking spermatids.

\section{Results}

\section{Population size in El Gallego and starting B frequency}

Applying the triple catch method (Begon 1979), and using the capture and recapture figures mentioned in the "Materials and methods" section, we estimated population size for each sex separately in El Gallego population. The results were 584 males and 645 females, indicating unbiased sex ratio in adults $\left(\chi^{2}=3.03, \mathrm{df}=1, P=0.082\right)$. Survival rates, calculated with these data showed values close to 1 in both sexes (0.944 in males and 1 in females). Therefore, the introduction of Salobreña males rendered an initial B frequency of 0.063 in males and 0.031 in the whole population of El Gallego.

\section{Evolution of B-chromosome frequency}

In 1993, i.e., one year before the release of males from the B-carrying population, we analyzed 82 individuals from El Gallego and 38 from Vicorto, and none of them carried B chromosomes (Cabrero et al. 1997). The analysis of the 1996 and 1999 samples showed the presence of B chromosomes in El Gallego, but not in Vicorto, for which reason 
we discontinued sampling in this latter population and concluded that B invasion failed in it. In El Gallego, however, the postrelease frequency of the B2 chromosome was equal to 0.031 in 1994 (see above), and we found one individual carrying B2 in 1996, but none later on (Table 1). However, from 1996 onwards, most samples showed a very low frequency of the B1 variant, with slight but not significant fluctuations across years (contingency test: $P=$ 0.112 , S.E. $=0.005$ ).

\section{Nature of the B chromosomes found in El Gallego across years}

We performed double FISH analysis with probes for the $45 \mathrm{~S}$ rDNA and a $180 \mathrm{bp}$ satDNA, in some males from the samples collected at four different years $(1996,1999,2008$,

Table 1 Evolution of B frequency in El Gallego across 20 years

\begin{tabular}{llllllll}
\hline & & & \multicolumn{2}{c}{$1 \mathrm{~B}$} & & \multicolumn{2}{l}{ Mean Bs } \\
Year & Sex & 0B & B2 & B1 & Total & B2 & B1 \\
\hline 1994 & Males & & & & & $0.031^{\mathrm{a}}$ & \\
1996 & Males & 46 & 1 & 2 & 49 & 0.020 & 0.041 \\
1999 & Males & 48 & 0 & 4 & 52 & 0 & 0.077 \\
2000 & Males & 49 & 0 & 0 & 49 & 0 & 0 \\
& Females & 44 & 0 & 3 & 47 & 0 & 0.064 \\
& Total & 93 & 0 & 3 & 96 & 0 & 0.031 \\
2002 & Males & 99 & 0 & 2 & 101 & 0 & 0.02 \\
& Females & 22 & 0 & 0 & 22 & 0 & 0 \\
& Total & 121 & 0 & 2 & 123 & 0 & 0.016 \\
2006 & Males & 65 & 0 & 0 & 65 & 0 & 0 \\
& Females & 12 & 0 & 0 & 12 & 0 & 0 \\
& Total & 77 & 0 & 0 & 77 & 0 & 0 \\
2008 & Males & 79 & 0 & 1 & 80 & 0 & 0.013 \\
Total & Males & 93 & 0 & 5 & 98 & 0 & 0.051 \\
\hline & & 557 & 1 & 17 & 575 & & \\
\hline
\end{tabular}

Note that the B2 chromosome carried by us from the Salobreña population was found in 1996 but not in subsequent samples. Also note that the B1 type is naturally found in downstream populations to El Gallego in the Segura River

${ }^{\mathrm{a}}$ Weighted B frequency estimated after mixing the B-carrying receptor population, whose size was estimated by the triple capture-recapture method, with the 50 males from Salobreña (with 0.80 B frequency) and 2013), as this technique allows to differentiate the B1 and $\mathrm{B} 2$ variants, the former being larger and bearing equal or higher amounts of rDNA and satDNA, whereas the latter carries higher amount of satDNA than rDNA (Fig. 1a, b). In 1996, we analyzed three B-carrying males by double FISH, and one of them carried the B2 chromosome, whereas each of the two other males carried one B1 chromosome. One of the B1-carrying males was homozygous (SS) for the presence of satDNA on autosome 9, whereas the two other males lacked satDNA on this chromosome (NN) (Fig. 1c). However, only B1 chromosomes were found in the males analyzed by double FISH in 1999 (14 B-lacking and one with 1B), 2008 (1 with 1B) and 2013 (68 B-lacking and five with 1B). This indicates that the introduced B2 chromosome remained in El Gallego population for at least two generations (up to 1996), but it was apparently absent from 1999 onwards, suggesting that the effect of B introduction had diluted in only a few years. Remarkably, the presence of satDNA-carrying autosome 9, which is typical of Salobreña males (Fig. 1a), showed a parallel dilution, since it was still found in 1999 but was absent later on (Fig. 1c). None of the 73 males analyzed by FISH in 2013 showed the presence of satDNA on autosome 9, confirming the disappearance of this chromosome marker.

\section{B-chromosome transmission}

In the 2000 sample, we analyzed in vivo the presence of B chromosomes in both males and females, and found no Bcarrying males but three females with one B1-type chromosome (Table 1). They were mated with $0 \mathrm{~B}$ males from the same population and two of them yielded eggs, i.e., two egg pods from one female and one egg pod from the other. The analysis of B-chromosome transmission through these two 1B females suggested a strong tendency to B elimination, with average transmission rate $\left(k_{B}=0.239\right)$ being significantly lower than the Mendelian one (0.5) (Table 2). In addition, we analyzed meiotic transmission of the B1 chromosome in five 1B males collected in 2013 at El Gallego population. We found significant $B$ elimination in two males, but average transmission rates for the five males ( 0.49 in $\mathrm{R}$ spermatids and 0.48 in elongating ones) were not significantly different from 0.5 (Table 3 ).
Table 2 Analysis of B1chromosome transmission through $1 \mathrm{~B}$ females mated with OB males

\begin{tabular}{llllllll}
\hline Bs in female & Eggs & Embryos & 0B embryos analyzed & 1B embryos analyzed & Total & $k_{B}$ & $Z$ \\
\hline 1 & 40 & 36 & 13 & 6 & 19 & 0.32 & -1.61 \\
1 & 34 & 33 & 22 & 5 & 27 & 0.19 & $-3.3^{*}$ \\
Total & 74 & 69 & 35 & 11 & 46 & 0.24 & $-7.1^{*}$ \\
\hline
\end{tabular}

$\mathrm{Z}$ is the approximation of binomial to normal distribution and its value indicates significant departure of Mendelian transmission rate $\left(k_{B}=0.5\right)$ when higher than 1.96 in absolute value, thus indicating $\mathrm{B}$ accumulation if positive or B elimination if negative (see "Methods")

$* P<0.05$ 


\section{Introgression of nuclear markers from Salobreña into El Gallego}

As an additional way of testing whether the Salobreña males released in 1994 actually mated resident females, we analyzed ISSR markers in 50 males from El Gallego in 2006 and compared their genotypes with those previously analyzed by us (Manrique-Poyato et al. (2013a, 2013b, 2015) in six populations collected, the same year, at Salobreña (the donor population, harboring B2-type chromosomes) and five populations geographically placed near El Gallego, i.e., Claras, Socovos, Caravaca, Mundo, and Calasparra, the three former lacking B chromosomes and the two latter carrying them (B1 type). Claras is located by the Taibilla River, a tributary of the Segura River, which drains to this latter upstream to El Gallego. Socovos and Caravaca are located by small streams draining to the Segura River, downstream to El

Table 3 Frequency of B-lacking (B-) and B1-carrying (B+) spermatids in five 1B males of the grasshopper E. plorans collected at El Gallego population in 2013

\begin{tabular}{lllllll}
\hline Male no. & Spermatid type & $\mathrm{B}-$ & $\mathrm{B}+$ & $k_{B}$ & $\chi$ & $P$ \\
\hline 2 & $\mathrm{R}$ & 44 & 62 & 0.58 & 3.06 & 0.0804 \\
& $\mathrm{E}$ & 34 & 19 & 0.36 & 4.25 & $0.0394^{*}$ \\
11 & $\mathrm{R}$ & 126 & 124 & 0.5 & 0.02 & 0.8993 \\
& $\mathrm{E}$ & 125 & 125 & 0.5 & 0 & 1 \\
48 & $\mathrm{R}$ & 122 & 97 & 0.44 & 2.85 & 0.0912 \\
& $\mathrm{E}$ & 170 & 162 & 0.49 & 0.19 & 0.6606 \\
55 & $\mathrm{R}$ & 47 & 24 & 0.34 & 7.45 & $0.0063^{* *}$ \\
& $\mathrm{E}$ & 29 & 18 & 0.38 & 2.57 & 0.1086 \\
78 & $\mathrm{R}$ & 122 & 128 & 0.51 & 0.14 & 0.7043 \\
& $\mathrm{E}$ & 127 & 124 & 0.49 & 0.04 & 0.8498 \\
\multirow{2}{*}{ Total } & $\mathrm{R}$ & 461 & 435 & 0.49 & 0.75 & 0.3851 \\
& $\mathrm{E}$ & 485 & 448 & 0.48 & 1.47 & 0.2258 \\
\hline
\end{tabular}

$R$ round, $E$ elongated, $k_{B}$ B transmission rate

$* P<0.05, * * P<0.01$
Gallego. Mundo is located by the Mundo River, north of the Segura River, and, in the analyzed sample, six out of sixteen males carried B chromosomes. Finally, Calasparra is located by the Segura River downstream to El Gallego and showed a low frequency of B chromosomes (two B-carrying out of forty-four males analyzed).

Our analysis yielded a total of 97 ISSR markers ranging in size from 180 to $2000 \mathrm{bp}$ in the six populations as a whole, with $99 \%$ of the loci being polymorphic. A single private allele was found (i.e., the 6-1600 allele), which was exclusive to El Gallego population. Calculation of pairwise Fst values (Table S1) revealed that El Gallego was the population showing the lowest differentiation in respect to Salobreña (the donor population), suggesting that the males released in 1994 at El Gallego actually mated and the effects were still apparent in 2006. Mantel test indicated significant positive correlation between pairwise Fst values and geographical distance $(r=0.84, P=0.0058)$, even when the Salobreña population was discarded $(r=0.49, P=0.0431)$, suggesting remarkable differentiation between populations next to El Gallego area, consistent with the genetic barriers previously detected in the Segura River basin (ManriquePoyato et al. 2015).

To analyze the presence of ISSR genotypes showing the profile of the donor population in El Gallego, we used the Structure software, which revealed that four genetic groups $(k=4)$ best fit the data (Table S2). Consistent with its most distant geographical location (232.5 km from El Gallego), the Salobreña population constituted group 1 (Fig. 2 and Table S3). The three other groups included ISSR genotypes from the Segura River basin, with group 2 being predominant in the three B-lacking populations placed near El Gallego (Claras, Socovos, and Caravaca), group 3 being mainly represented by El Gallego individuals, and group 4 being best represented in the two B-carrying populations from the Segura River basin (Mundo and Calasparra), but being also frequent (at a lower degree) in El Gallego, Claras, and Socovos. Remarkably, El Gallego showed the

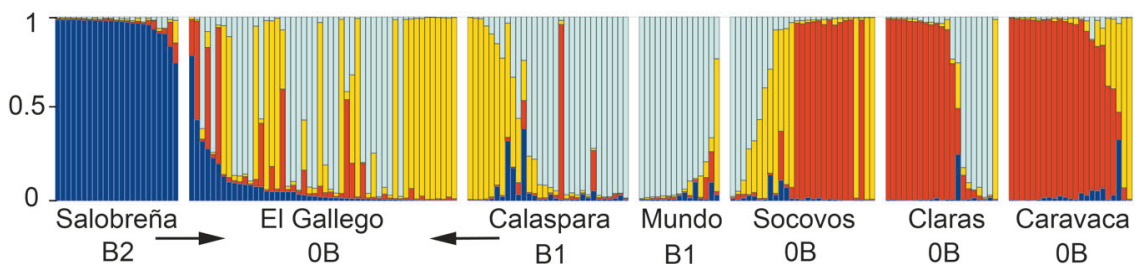

Fig. 2 ISSR markers analyzed in 2006 in seven natural populations. Each multicolored vertical bar represents the ancestry of each of the 189 individuals analyzed, for the four genetic groups $(K=4)$ yielded by the Structure software using the ISSR markers. To get a more illustrative diagram, individuals within each population were sorted to show decreasing ancestry for group 1 (Salobreña and El Gallego), group 2 (Claras and Caravaca), group 3 (Calasparra), or group 4 (Mundo and Socovos). Note that group 1 ancestry (dark blue) in El
Gallego reflects the effect of the forced introgression of Salobreña males in 1994 (arrow on the left). Likewise, the high ancestry for groups 3 and 4 (yellow and cyan, respectively) in El Gallego reflects the recurrent introgression from B1-carrying populations, forced by fishermen (arrow on the right). The absence of B chromosomes in the populations is indicated as $\mathrm{OB}$ (in El Gallego, it refers to a sample prior to 1994), whereas B chromosome presence is indicated by the B type, i.e., $\mathrm{B} 1$ or $\mathrm{B} 2$ 
highest ancestry with the Salobreña group, specifically being about 1.5-3.8 times higher than those observed in the remaining five populations from the Segura River basin (Table S3). As shown in Fig. 2 and Table S4, one individual from El Gallego showed ISSR profiles being $75 \%$ similar to those found in Salobreña. Taken together, these results indicate that the Salobreña males introduced in 1994 by us in El Gallego successfully mated, and the genetic effects were still apparent in 2006, although highly diluted.

On the other hand, Table S3 and Fig. 2 show that El Gallego population showed about the same ancestry with groups 3 and 4, the latter including most individuals from Calasparra and Mundo populations. Remarkably, these two populations belong to the B-carrying area in the Segura River basin and might constitute the main sources for the arrival of B1-type chromosomes to El Gallego, transported by fishermen, as discussed below.

\section{Discussion}

The B chromosome system in the grasshopper E. plorans is a paradigm of the coevolution between $\mathrm{A}$ and $\mathrm{B}$ chromosomes. These harmful B chromosomes invade populations because of their drive through females, although this is rapidly suppressed by the host genome (Perfectti et al 2004). According to the near-neutral model of B chromosome evolution (Camacho et al. 1997), B chromosome invasions are expected to be rapid prior to the action of drive-suppressor genes, as drive increases B frequency quickly. We have evidenced several natural invasions of B chromosomes in E. plorans, supporting this prediction. We first observed that the mean number of B24 chromosomes per individual, in the Torrox population, increased from 0.34 in 1984 till 1 in 1992 and 1.54 in 1994, with B24 drive being close to 0.7 in 1992 (Zurita et al. 1998). The second invasion took place in Pollensa (Mallorca), where B1 frequency increased one order of magnitude (from 0.053 to 0.692 ) in only 10 years (Riera et al. 2004). The third invasion was registered in Otivar, where B2 frequency increased from 0.26 to 0.57 between 1977 and 1992, and had raised till 0.86 in 2012 (Camacho et al. 2015). Such a rapid increase in $\mathrm{B}$ frequency was thus the expectation for a successful invasion in El Gallego and Vicorto. However, we did not find B chromosomes in Vicorto 2 and 5 years later to the release of Salobreña males, suggesting that B2 invasion in this population failed since the very beginning. In El Gallego, however, we found the B2 chromosome in 1996 ( 2 years after males release), along with the B1 variant, but the latter was the only B type found in subsequent samples, although at very low frequency. This indicates that the 1994 release of Salobreña males was effective in the short term, as is also suggested by the presence of the
satDNA on autosome 9 in the 1996 and 1999 samples. However, the B2 chromosome and the autosomal marker were not observed in subsequent samples, indicating the dilution of the donor population markers onto the receptor population. The fact that population size was high in the receptor population implied a very low starting frequency for donor markers (e.g., 0.031 for B2) and thus high likelihood of disappearance by drift. An alternative explanation for such a fast B2 elimination is that the receptor populations already contained drive suppressors due to previous B chromosome presence, as the recurrent forced migration of B1-carrying males by fishermen might suggest (see below).

Prior to starting the experiment, in 1994, we had performed experimental crossings that showed the absence of drive through both sexes (López-León et al. 1992). For this reason, and in order to exert minimum impact on the donor population (as female is the sex limiting fecundity), we opted for introducing males. However, only 2 years after El Gallego and Vicorto artificial introductions, we learned that 1B females from Salobreña can show drive when mated to males from a B-lacking population (Herrera et al. 1996), so that female is the driving sex in E. plorans. This indicates that the introduction of Salobreña females would have had higher likelihood for a successful B2 invasion. As shown by computer simulation analyses, in the absence of drive or beneficial effects on B-carriers, a B chromosome cannot invade a population (Camacho et al. 1997). This predicted a low likelihood of successful B2 invasion in El Gallego, since we had introduced individuals of the nondriving sex, and it was what happened with B2 and satDNA on autosome 9 being diluted in only a few generations. The ISSR analysis showed evidence for the presence of donor genotypes still in 2006, although at very low frequency (only one individual from El Gallego presented ISSR markers being $75 \%$ similar to those in Salobreña).

Gosálvez and López-Fernández (1984) performed a similar experiment in the grasshopper Arcyptera fusca. They selected a B-lacking population in Puerto de la Bonaigua (Valle de Arán, Lérida, Spain) and released, for two consecutive years (1978 and 1979), 50 young males from a B-carrying population located at Valle del Respumoso (130 km distance), where about $50 \%$ of individuals carried B chromosomes across generations (López-Fernández and Gosálvez 1981; 1983; López-Fernández et al. 1983). No B-carrying males were found 1 year after the first release and only two B-carrying males were captured 1 year after the second release, with all the individuals subsequently collected (in 1981 and 1982) lacking B chromosomes (Gosálvez and López-Fernández 1984). These authors concluded that the introduced B chromosomes had been eliminated.

Unexpectedly, in five out of the six E. plorans samples analyzed in El Gallego between 1996 and 2013 we found a 
B chromosome that, on the basis of size and proportions of satDNA and rDNA (Cabrero et al. 2014), belongs to the B1type characteristic of populations in the Segura River located downstream to El Gallego, such as Mundo and Calasparra, sited at only 20 and $33 \mathrm{~km}$ distance, respectively (see Table S1). This suggests the existence of migration to El Gallego from B1-carrying populations downstream, which is independent of our experimental release of B2carrying males from Salobreña. During our 2013 collection trip, we witnessed the use of E. plorans males as bait for recreational fishing of rainbow trout (Oncorhynchus mykiss) around El Gallego. During the 2013 sampling, we met two fishermen in El Gallego, who each carried a plastic bottle with some rice branches and 20-25 E. plorans males to use them as bait. They said that those grasshoppers had been captured in Calasparra (33 km away), in the same rice crops in which we had previously collected samples of $E$. plorans that harbor the B1 chromosome. The fishermen also told us that when they finished fishing, the leftover grasshoppers were released in situ, which suggests a pathway for recurrent forced migration of E. plorans males to El Gallego. The large size of E. plorans females makes them useless as bait for rainbow trout. Therefore, the low B1 frequency observed by us across years in El Gallego population indicates that recurrent forced migration of $E$. plorans males, performed by fishermen, is also an unlikely means to successful invasion of the B1 chromosome onto the Blacking region. Indirect evidence for forced migration of $E$. plorans by fishermen is the higher ISSR ancestry of El Gallego with groups 3 and 4 populations, i.e., those belonging to the B-carrying region of the Segura River basin (Mundo and Calasparra). This possibility is also supported by the coincidence of the highest fishing activity (July and August) with the highest abundance of E. plorans in the zone.

We favor a model where the unsuccessful B2 chromosome invasion in El Gallego was due to the introduction of males, which transmit B chromosomes at Mendelian rates (López-León et al. 1992), whereas these B chromosomes drive only through females (Herrera et al. 1996; Zurita et al. 1998). For the same reason, the recurrent introduction of $E$. plorans males from populations carrying B1 chromosomes by fishermen is also useless for B1 invasion, as they do not use females for fishery. Intriguingly, we observed very low B1 transmission rate through $\mathrm{B} 1$-carrying females from El Gallego. A possible explanation is that the $\mathrm{B}$ chromosome drive is rapidly suppressed in this species (Perfectti et al. 2004), and the B1 chromosome in other populations like Daimuz (Valencia, Spain) does not show drive (LópezLeón et al. 1992). The fact that B chromosomes in $E$. plorans only drive through females implies that releasing males postpones one generation the possibility of B drive, i.e., until mating of hybrid females in the next generation. However, both the low B-transmission rates shown by one B1 females in 2000 and B2 disappearance in only a few years, indicate that hybrid females did not manifest important B chromosome drive, perhaps due to its rapid suppression. The possibility that B2 was outcompeted by B1 can also be ruled out since both B types were never seen in a same individual from El Gallego population and failed to increase in frequency across years (see Table 1). Therefore, additional research is needed to solve this conundrum.

We report here two examples of migration that failed to lead to B-chromosome invasion. Our single 1994 release caused a genetic impact in the receptor population, but it was rapidly diluted in subsequent years. However, the recurrent forced male migration by fishermen is producing a genetic impact, which makes El Gallego population more genetically similar to B-carrying populations, like El Mundo and Calasparra, than to B-lacking ones geographically sited closer to it (like Socovos) (see Table S1). In both types of migration events the B2 and B1 chromosomes, respectively, failed to increase in frequency across years. It is thus tempting to speculate that an effective Bchromosome invasion would have needed drive from the very beginning, as predicted by the near-neutral model (Camacho et al. 1997), and that perhaps the result of our experiment would have been different if we had released females rather than males. Our present results thus suggest that B-chromosome invasions are probably more effective by migration of individuals from the sex where B chromosomes show drive, i.e., the female in E. plorans. The B2 transmission rate expected for Salobreña females mated with El Gallego males (if we would have released females) should be similar to that shown by Salobreña females crossed to males from Claras (a B-lacking population sited $20 \mathrm{~km}$ upstream in the Segura river), which showed B2 transmission rates significantly higher than the Mendelian one (Herrera et al. 1996). Unfortunately, in 1994, we did not know this when released Salobreña males in El Gallego. However, it is still intriguing why putative B2carrying females born in 1995 did not show drive enough to increase B2 frequency in the receptor population. A plausible explanation could come in the near future through the analysis of the genetic and epigenetic changes in gene expression that probably occur after interpopulation crosses. This kind of research is now feasible since the gene content of these $\mathrm{B}$ chromosomes begins to be known (Navarro-Domínguez et al. 2017).

\section{Data archiving}

ISSR data available from Dryad: https://doi.org/10.5061/ dryad.g3h1444. 


\section{Data availability}

All data about B-chromosome frequency and transmission are available in the manuscript and supplementary material, and ISSR marker genotypes are available in Dryad.

Acknowledgements We thank the Consejería de Agricultura of the Junta de Comunidades de Castilla-La Mancha for giving us the permit to release E. plorans grasshoppers from Salobreña at El Gallego and Vicorto populations. We also thank an anonymous reviewer whose helpful suggestions improved the manuscript. This study was supported by grants from the Spanish Secretaría de Estado de Investigación, Desarrollo e Innovación (CGL2015-70750-P), and was partially performed by FEDER funds.

\section{Compliance with ethical standards}

Conflict of interest The authors declare that they have no conflict of interest.

Publisher's note: Springer Nature remains neutral with regard to jurisdictional claims in published maps and institutional affiliations.

\section{References}

Bakkali M, Camacho JPM (2004) The B chromosome system of the grasshopper Eyprepocnemis plorans in North Africa. IV. Transmission of rare B chromosome variants. Cytogenet Genome Res 106:332-337

Bakkali M, Perfectti F, Camacho JPM (2002) The B chromosome polymorphism of the grasshopper Eyprepocnemis plorans in North Africa. II. Parasitic and neutralized B chromosomes. Heredity $88: 14-18$

Begon M (1979) Investigating animal abundance: capture-recapture for biologists. Edward Arnold Publishers, London

Cabrero J, López-León MD, Gómez R, Castro AJ, Martín-Alganza A, Camacho JPM (1997) Geographical distribution of B chromosomes in the grasshopper Eyprepocnemis plorans, along a river basin, is mainly shaped by non-selective historical events. Chromosome Res 5:194-198

Cabrero J, López-León MD, Ruíz-Estévez M, Gómez R, Petitpierre E, Rufas JS, Massa B, Ben Halima MK, Camacho JPM (2014) B1 was the ancestor B chromosome variant in the western Mediterranean area in the grasshopper Eyprepocnemis plorans. Cytogenet Genome Res 142:52-58

Cabrero J, Manrique-Poyato MI, Camacho JPM (2006) Detection of B chromosomes in interphase hemolymph nuclei from living specimens of the grasshopper Eyprepocnemis plorans. Cytogenet Genome Res 114:66-69

Cabrero J, Martín-Peciña M, Ruiz-Ruano FJ, Gómez R, Camacho JPM (2017) Post-meiotic B chromosome expulsion, during spermiogenesis, in two grasshopper species. Chromosoma 126:633-644

Cabrero J, Perfectti F, Gómez R, Camacho JPM, López-León MD (2003) Population variation in the A chromosome distribution of satellite DNA and ribosomal DNA in the grasshopper Eyprepocnemis plorans. Chromosome Res 11:375-381

Camacho JPM, Cabrero J, López-León MD, Bakkali M, Perfectti F (2003) The B chromosomes of the grasshopper Eyprepocnemis plorans and the intragenomic conflict. Genetica 117:77-84

Camacho JPM, Shaw MW, Cabrero J, Bakkali M, Ruíz-Estévez M, Ruíz-Ruano FJ, Martín-Blázquez R, López-León MD (2015) Transient microgeographic clines during $\mathrm{B}$ chromosome invasion. Am Nat 186:675-681
Camacho JPM, Shaw MW, López-León MD, Pardo MC, Cabrero J (1997) Population dynamics of a selfish B chromosome neutralized by the standard genome in the grasshopper Eyprepocnemis plorans. Am Nat 149:1030-1050

Darlington CD (1958) Evolution of genetic systems. Oliver and Boyd, Edinburgh and London

Dirsh VM (1958) Revision of the genus Eyprepocnemis Fieber, 1893 (Orthoptera: Acridoidea). Proc R Entomol Soc Lond B 27:33-45

Earl DA, VonHoldt BM (2012) Structure harvester: a website and program for visualizing STRUCTURE output and implementing the Evanno method. Conserv Genet Resour 4:359-361

Evanno G, Regnaut S, Goudet J (2005) Detecting the number of clusters of individuals using the software structure: a simulation study. Mol Ecol 14:2611-2620

Falush D, Stephens M, Pritchard JK (2007) Inference of population structure using multilocus genotype data: dominant markers and null alleles. Mol Ecol Notes 7:574-578

Gosálvez J, López-Fernández C (1984) Elimination of an introduced B-chromosome from a wild population of Arcyptera fusca (Orthoptera). Heredity 53:453-456

Herrera JA, López-León MD, Cabrero J, Shaw MW, Camacho JPM (1996) Evidence for B chromosome drive suppression in the grasshopper Eyprepocnemis plorans. Heredity 76:633-639

Hewitt GM (1976) Meiotic drive for B chromosomes in the primary oocytes of Myrmeleotettix maculatus (Orthoptera: Acrididae). Chromosoma 56:381-391

Holsinger KE, Lewis PO, Dey DK (2002) A Bayesian method for analysis of genetic population structure with dominant marker data. Mol Ecol 11:1157-1164

Jones RN (1991) B-chromosome drive. Am Nat 137:430-442

Lanzas P, Perfectti F, Garrido-Ramos MA, Ruíz-Rejón C, GonzálezSánchez M, Puertas M, Camacho JPM (2018) Long-term monitoring of B-chromosome invasion and neutralization in a population of Prospero autumnale (Asparagaceae). Evolution $72: 1216-1224$

López-Fernández C, García de la Vega C, Gosálvez J (1983) Spontaneous supernumerary isochromosomes in the grasshopper Arcyptera fusca (Pall). Cytobios 37:107-111

López-Fernández C, Gosálvez J (1981) Differential staining of a heterochromatic zone in Arcyptera fusca (Orthoptera). Experientia $37: 240$

López-Fernández C, Gosálvez J (1983) The chromosome system in three species of the genus Arcyptera (Orthoptera: Acrididae). II. Unstable B-chromosomes in Arcyptera fusca. Genetica 62:41-46

López-León MD, Cabrero J, Camacho JPM, Cano MI, Santos JL (1992) A widespread B chromosome polymorphism maintained without apparent drive. Evolution 46:529-539

López-León MD, Cabrero J, Dzyubenko VV, Bugrov AG, Karamysheva TV, Rubtsov NB, Camacho JPM (2008) Differences in ribosomal DNA distribution on $\mathrm{A}$ and $\mathrm{B}$ chromosomes between eastern and western populations of the grasshopper Eyprepocnemis plorans plorans. Cytogenet Genome Res 121:260-265

López-León MD, Pardo MC, Cabrero J, Viseras E, Camacho JPM, Santos JL (1993) Generating high variability of B chromosomes in the grasshopper Eyprepocnemis plorans. Heredity 71:352-362

Manrique-Poyato MI, López-León MD, Cabrero J, Gómez R, Perfectti F, Camacho JPM (2015) Geographical barriers impeded the spread of a parasitic chromosome. PLoS ONE 10:e0131277

Manrique-Poyato MI, López-León MD, Cabrero J, Perfectti F, Camacho JPM (2013a) Spread of a new parasitic B chromosome variant is facilitated by high gene flow. PLoS ONE 8:e83712

Manrique-Poyato MI, López-León MD, Gómez R, Perfectti F, Camacho JPM (2013b) Population genetic structure of the grasshopper Eyprepocnemis plorans in the south and east of the Iberian Peninsula. PLoS ONE 8:e59041 
Muñoz-Pajares AJ, Martínez-Rodríguez L, Teruel M, Cabrero J, Camacho JPM, Perfectti F (2011) A single, recent origin of the accessory B chromosome of the grasshopper Eyprepocnemis plorans. Genetics 187:853-863

Navarro-Domínguez B, Ruiz-Ruano FJ, Cabrero J, Corral JM, LópezLeón MD, Sharbel TF, Camacho JPM (2017) Protein-coding genes in $\mathrm{B}$ chromosomes of the grasshopper Eyprepocnemis plorans. Sci Rep 7:45200

Nur U (1962) A supernumerary chromosome with an accumulation mechanism in the lecanoid genetic system. Chromosoma 13:249-271

Nur U, Brett BLH (1985) Genotypes suppressing meiotic drive of a B chromosome in the mealy bug Pseudococcus obscurus. Genetics 110:73-92

Östergren G (1945) Parasitic nature of extra fragment chromosomes. Bot Not 2:157-163

Pardo MC, López-León MD, Cabrero J, Camacho JPM (1994) Transmission analysis of mitotically unstable B chromosomes in Locusta migratoria. Genome 37:1027-1034
Perfectti F, Corral JM, Mesa JA, Cabrero J, Bakkali M, López-León MD, Camacho JPM (2004) Rapid suppression of drive for a parasitic B chromosome. Cytogenet Genome Res 106:338-343

Riera L, Petitpierre E, Juan C, Cabrero J, Camacho JPM (2004) Evolutionary dynamics of a B-chromosome invasion in island populations of the grasshopper Eyprepocnemis plorans. J Evol Biol 17:716-719

Shaw MW (1984) The population genetics of the B-chromosome polymorphism of Myrmeleotettix maculatus (Orthoptera: Acrididae). Biol J Linn Soc 23:77-100

Shaw MW, Hewitt GM (1985) The genetic control of meiotic drive acting on the B chromosome of Myrmeleotettix maculatus (Orthoptera: Acrididae). Heredity 54:259-268

White MJD (1973) Animal Cytology and Evolution. 3rd edn. Cambridge University Press, London

Zurita S, Cabrero J, López-León MD, Camacho JPM (1998) Polymorphism regeneration for a neutralized selfish B chromosome. Evolution 52:274-277 